\title{
Global Sausage Magnetoacoustic Modes of Coronal Loops
}

\author{
V.M. Nakariakov ${ }^{1}$, V.F. Melnikov ${ }^{2}$ \\ and V.E. Reznikova ${ }^{2}$ \\ ${ }^{1}$ Physics Department, University of Warwick, Coventry, CV4 7AL, email: \\ valery@astro.warwick.ac.uk \\ ${ }^{2}$ Radiophysical Research Institute (NIRFI), Nizhny Novgorod, 603950, Russia, email: \\ meln@nirfi.sci-nnov.ru, reznik@nirfi.sci-nnov.ru
}

\begin{abstract}
A sufficiently thick and dense flaring magnetic loop is shown to be able to support global sausage magnetoacoustic modes. The oscillation period of this mode, calculated in the straight cylinder approximation, is determined by the length of the loop, not by its diameter, as it has previously been assumed. For the existence of this mode, the ratio of the loop length to its diameter is to be less than about a half of the square root of the density contrast ratio. This mode has a maximum of the magnetic field perturbation at the loop apex and nodes at the footpoints. We demonstrate that the 14-17 sec quasi-periodical pulsations of microwave emission, oscillating in phase at a loop apex and at its legs, as observed with the Nobeyama Radioheliograph, are interpreted in terms of the global sausage mode.
\end{abstract}

\section{Introduction}

The MHD sausage mode of coronal loop has been used to interpret periodicities in the range $0.5-5 \mathrm{~s}$, which are usually observed as modulation of coronal radio emission. The estimation of the periodicity has been based upon the assumption that the period of the sausage mode was determined by the ratio of the loop cross-section radius and the Alfvén speed inside the loop (see, e.g. Aschwanden 1987).

It has been pointed out by Roberts, Edwin \& Benz (1983) (see also Edwin \& Roberts 1983) that the estimation could be used for higher spatial harmonics only. Unfortunately, the consequent papers usually did not mention this crucial restriction. Also, this estimation could not be applied to the period of global sausage mode.

\section{The period of a global sausage mode}

In the paper by Nakariakov et al. 2003 we demonstrate that the expression traditionally used for the estimations of the sausage mode period

$$
P_{\text {saus }}=2 \pi a / C_{A 0},
$$

(where $a$ is the loop cross-section radius and $C_{A 0}$ is the Alfvén speed inside the loop) was over-simplified.

The correct period $P_{\mathrm{GSM}}$ of the global sausage mode of a coronal loop is determined as follows

$$
P_{\mathrm{GSM}}=2 L / C_{p},
$$

where $C_{p}$ is the phase speed of the sausage mode corresponding to the wave number $k=\pi / L, C_{A 0}<C_{p}<C_{A e}, C_{A e}$ is the Alfvén speed outside the loop. This mode has a maximum of the magnetic field perturbation at the loop apex and nodes at the 
footpoints. In addition, for a strong density contrast inside and outside the loop, the period of the sausage mode must satisfy the condition

$$
P_{\mathrm{GSM}}<\frac{2 \pi a}{j_{0} C_{A 0}} \approx \frac{2.62 a}{C_{A 0}} .
$$

We would like to stress that expression (2.3) is an inequality, and that the actual resonant frequency is determined by Eq. (2.2), provided (2.3) is satisfied. Combining (2.3) and (2.2), we obtain that the necessary condition for the existence of the global sausage mode is

$$
L / 2 a<\pi C_{A e} / 2 j_{0} C_{A 0} \approx 0.65 \sqrt{\rho_{0} / \rho_{e}},
$$

so the loop should be sufficiently thick and dense.

\section{Observation of a global sausage mode}

The observational proof of the existence of the global sausage mode should be based upon the determination of the oscillation period, the longitudinal and transverse sizes of the magnetic loop, and the spatial distribution of the oscillation amplitude along the loop. For this purpose we need an instrument that is able to provide us with well resolved images and a good enough time resolution. Nowadays, a necessary spatial (10"-5") and temporal $(0.1 \mathrm{~s})$ resolution is provided by the Nobeyama Radioheliograph (NoRH) operating at the high microwave frequencies 17 and $34 \mathrm{GHz}$ (Nakajima et al. 1994).

A good candidate for such a proof is a solar flare which happened on the 12th of January, 2000, and observed by NoRH at both frequencies. Melnikov, Reznikova \& Shibasaki (2002) found that the time profiles of microwave emission at 17 and $34 \mathrm{GHz}$ of this flare exhibited quasi-periodic $\left(P_{1}=14-17 \mathrm{~s}\right.$ and $\left.P_{2}=8-11 \mathrm{~s}\right)$ variations of the intensity, synchronous in different parts (near the looptop and footpoints) of the loop. The length of the flaring loop is estimated as $L=25 \mathrm{Mm}(\approx 34 ")$ and its width at half intensity at $34 \mathrm{GHz}$ as about $6 \mathrm{Mm}(\approx 8 ")$. The loop is estimated to be filled by a dense plasma with the concentration $n_{0} \approx 10^{17} \mathrm{~m}^{-3}$ penetrated by the magnetic field of the strength $B_{0} \approx 50-100 \mathrm{G}$. The detailed analysis shows that all these observed parameters well agree with the simultaneous existence of two sausage modes of oscillations in the loop: the global one $\left(P_{1}=14-17 \mathrm{~s}\right)$, with the maximum at the loop apex and the nodes at the footpoints, and a spatial harmonic, with $P_{2}=8-11$ s.

\section{Acknowledgements}

The work was partly supported by the RFBR grants No.02-02-39005, 04-02-16753 and by the Royal Society British-Russian Research Collaboration grant.

\section{References}

Aschwanden M. J. 1987 Sol. Phys. 111, 113.

Edwin P. M., Roberts B. 1983, Sol. Phys. 88, 179.

Melnikov V.F, Reznikova V.E., \& Shibasaki K. 2002 Phys. Proc. of Intern. Conf. "Active processes on the Sun and stars", Eds. Zaitsev V.V. and Yasnov L.V., 225.

Melnikov V.F., Reznikova V.E., Shibasaki K., \& Nakariakov V.M. 2004 these Proceedings.

Nakajima H. et al. 1994 The Nobeyama Radioheliograph. Proc. of the IEEE., 82, 705.

Nakariakov V.M., Melnikov V.F., Reznikova V.E. 2003 A $ध A$ 412, L7.

Roberts B., Edwin P.M., Benz, A.O. 1983 Nature, 305, 688. 